\title{
Desenvolvimento de um dispositivo de fixação baseado em diretrizes de projeto para confiabilidade
}

\section{Development of a fixture based on design guidelines for reliability}

\section{Resumo}

Em operações de usinagem, uma das etapas essenciais é a fixação das peças. Essa operação é comumente realizada por meio de dispositivos que compõem os equipamentos. Geralmente, em casos em que se necessita realizar o reposicionamento dessas peças, esses dispositivos utilizados não são suficientes para garantir requisitos técnicos relacionados às tolerâncias dimensionais e geométricas, o que acarreta dificuldades no processo e prejuízos para as empresas. A partir dessa perspectiva, este trabalho tem como objetivos desenvolver, construir e testar um dispositivo de fixação para ser utilizado em operações de usinagem ou medições permitindo reposicionamentos com garantia de qualidade dimensional e geométrica. Para isso, será abordado o processo de desenvolvimento de produtos, alinhado aos conceitos

Cassiano Rodrigues

\section{Moura}

cassiano.moura@ifsc.edu. br

Instituto Federal de Santa Catarina (IFSC)

Giovani Conrado Carlini giovani.carlini@ifsc.edu.br Instituto Federal de Santa Catarina (IFSC)

Cristiano da Silva iD cristiano.silva@ifsc.edu.br Instituto Federal de Santa Catarina (IFSC) de DfX - Design for Excellence (Projeto para Excelência), especificamente o projeto para confiabilidade, a fim de garantir um projeto de acordo com as premissas preestabelecidas. A metodologia utilizada neste trabalho caracteriza-se por meio de pesquisa tecnológica, que busca a geração de novos produtos, iniciando pela definição do projeto a partir de concepção preliminar, na qual são apresentadas as diretrizes de construção do dispositivo, bem como a descrição de seu projeto conceitual. Na sequência, é realizado o projeto detalhado, no qual são apresentadas as configurações específicas de cada componente do produto, a sua montagem e os materiais utilizados. Por fim, é realizada uma análise criteriosa, baseada em testes e ensaios, para validar e aprovar a construção do produto. Os resultados se mostraram coerentes com o objetivo proposto, sendo o produto apresentado validado pelos testes e aprovado conforme os critérios essenciais de aceitação do dispositivo.

Palavras-chave: DfX; Projeto para confiabilidade; Desenvolvimento de Produto; Dispositivo de fixação; Usinagem.

\begin{abstract}
In machining operations, one of the essential steps is to fix the parts. This operation is commonly performed through devices that make up the equipment. Generally, in cases where it is necessary to perform the repositioning of these parts, these devices used are not sufficient to guarantee technical requirements related to dimensional and geometric tolerances, which causes difficulties in the process and losses for companies. From this perspective, this work aims to develop, build and test a clamping device to be used in machining operations or measurements, allowing repositioning with guaranteed dimensional and geometric quality. For this, the Product Development Process will be approached, aligned with the concepts of DfX - Project for Excellence, specifically the project for reliability, in order to guarantee a project according to the pre-established premises. The methodology used in this work is characterized by technological research, which seeks the generation of new products, starting with the definition of the project through preliminary design, where the device construction guidelines are presented, as well as the description of its conceptual project. Following, the detailed project is carried out, where the specific configurations of each product component, its assembly and materials used are presented. Finally, a careful analysis based on tests and trials is carried out to validate and approve the construction of the product. The results were consistent with the proposed objective, and the product presented was validated by the tests and approved according to the essential criteria for acceptance of the device.
\end{abstract}

Keywords: DfX; Design for Reliability; Product Development; Fixture; Machining. 


\section{Introdução}

Durante os processos de usinagem e suas diferenciadas alternativas para confecção de peças, seja por meio de geometria definida, processos abrasivos, seja por métodos não convencionais (ZHAO, 2019), a fixação das peças é uma etapa de extrema importância para a confecção do produto ou componente dentro das especificações de projeto, observando atenciosamente os requisitos de segurança no ambiente de trabalho.Muitas operações de usinagem requerem acessórios para que as peças tenham posicionamento e fixação adequados em máquinas-ferramentas, principalmente do tipo comando numérico computadorizado (CNC). Para alcançar altos níveis de precisão de posicionamento da peça em relação à ferramenta, o dispositivo geralmente fornece contato com algumas superfícies de referência da peça. Tradicionalmente, é necessária alta precisão para a fabricação e montagem desses dispositivos para garantir a exatidão do posicionamento da peça (LIU; LI; WEN, 2019). Atualmente, na literatura, existem abordagens para o desenvolvimento em posicionar os componentes por intermédio de um sistema mecânico baseado em abordagens analíticas e simulações numéricas (IVANOV et al., 2019). A utilização de realidade aumentada e de sistemas de inteligência artificial também colabora para o desenvolvimento de novas soluções (TZIMAS; VOSNIAKOS; MATSAS, 2019). Geralmente, após a fixação da peça e iniciado o processo de usinagem, este deve ser finalizado para, então, se retirar ou deslocar a peça de sua posição original. Em alguns casos, quando se faz necessário deslocar ou mover a peça antes de se finalizar a usinagem, pode-se comprometer algumas tolerâncias geométricas, como cilindricidade e posicionamento, entre outras, gerando erros que podem afetar negativamente o desempenho dessas peças, inviabilizando-as, o que impacta diretamente nos custos das empresas. As peças especificadas como rotacionais axissimétricas e as classes de componentes prismáticos correspondem aos sistemas de fixação simples e tradicionais (KARPUS et al., 2019). Na maioria dos casos, essa fixação é realizada por meio de morsas, placas universais, grampos ou fixadores localizados no próprio equipamento. Embora uma gama de componentes apresenta formas complexas de geometria, partes dessa classe são caracterizadas pela complexidade das configurações de fixação resultante da complexa relação espacial das superfícies (IVANOV; ZAJAC, 2018). Nesse contexto, os dispositivos de fixação utilizados em operações de usinagem geralmente não são suficientes para garantir tolerâncias geométricas em casos de reposicionamento de peças. Assim, soluções organizacionais e funcionais que agilizem todo o ciclo de projeto e produção são extremamente valiosos nesse tipo de produto. Assim, para seguir as tendências do mercado e aumentar a competitividade, vários projetos utilizam a metodologia Design for Excellence (DfX), uma ferramenta que procura aprimorar os projetos de desenvolvimento de produtos em diversas vertentes, buscando encontrar uma solução de compromisso otimizada, na perspectiva quantitativa, para a melhor assertividade do componente (MENDES et al., 2019).Diante disso, este trabalho tem como objetivo desenvolver, construir e testar um dispositivo de fixação para usinagem utilizando técnicas do processo de desenvolvimento de produtos alinhado aos conceitos de $\mathrm{DfX}$ - Projeto para excelência, a fim de projetar um equipamento que possa garantir a confiabilidade e a qualidade do sistema de fixação.

\section{Revisão bibliográfica}

\subsection{Processo de desenvolvimento de produtos}

O processo de desenvolvimento de produto (PDP) pode ser entendido como um conjunto de atividades por meio das quais se pode chegar às especificações do projeto de um produto ou de seu processo de produção, para que a manufatura seja capaz de produzi-lo (ROZENFELD et al., 2006). Diversos autores, como Medeiros (1981), Buss e Cunha (2002), Rozenfeld et al. (2006) e Pahl et al. (2013), definem métodos pra desenvolver projetos como meios para o produto atender à necessidade do mercado, com qualidade e tempo adequado, a um custo compatível e garantindo a manufaturabilidade do produto.

De acordo com Rozenfeld et al. (2006), um bom modelo de desenvolvimento pode ajudar a materializar as políticas e estratégias gerenciais e racionalizar o fluxo de informações e de documentos durante o desenvolvimento de produtos, contribuindo para a integração da empresa em torno de uma visão única e focalizada num tipo de 
negócio, direcionando-a para um determinado mercado ou cliente. O autor faz uma comparação com outros processos de negócio, destacando como principais diferenças:

- Elevado grau de incerteza e risco das atividades;

- Decisões importantes são tomadas no início;

- Dificuldade em se mudar decisões iniciais;

- As atividades seguem um ciclo iterativo;

- Manipulação e geração de um alto volume de informação;

- As informações provêm de diversas fontes e áreas da empresa.

Para se desenvolver um produto de forma eficiente é necessário um processo eficaz para cumprir com os objetivos da organização, favorecendo a competitividade da empresa. Esse desempenho está diretamente relacionado com o modelo de desenvolvimento adotado, que, por sua vez, determina a capacidade com que as empresas lidam com os processos e a forma de interação com o mercado e as fontes de tecnologias. Pahl et al. (2013) apresentaram um modelo para o processo de desenvolvimento de projeto, que pode ser desdobrado nas seguintes fases:

- Planejar e esclarecer a tarefa (definição informativa);

- Conceber (definição preliminar);

- Projetar (definição da configuração);

- Detalhar (definição da tecnologia de produção).

\subsection{Design for Excellence - DfX}

Conforme apresentado por Rozenfeld et al. (2006), o processo de desenvolvimento de produtos é uma tarefa complexa que pode apresentar diversas dificuldades durante sua aplicação, principalmente nas primeiras fases, em que as principiais decisões devem ser tomadas.

Para auxiliar o projetista nessa tarefa, existem ferramentas que podem ser aplicadas durante o processo para se aumentar as possibilidades de sucesso, entre elas pode-se citar a abordagem DfX, que consiste em uma técnica baseada em princípios, orientações, recomendações e regras para auxiliar o projeto do produto de forma a facilitar sua fabricação ou montagem (BRALLA, 1996). Na literatura, são apresentados diversos métodos envolvendo $D f X$, conforme pode-se observar na Tabela 1.

Tabela 1 - Variações da metodologia $D f X$.

\begin{tabular}{ll}
\hline Design for Aesthetics & Projeto para Estética \\
Design for Assembly & Projeto para Montagem \\
Design for Configuration & Projeto para Configuração \\
Design for Cost / Profit & Projeto para Custo \\
Design for Environment & Projeto para Meio Ambiente \\
Design for Inspectability & Projeto para Inspeção \\
Design for Mantainability & Projeto para Mantenabilidade \\
Design for Manufacturing & Projeto para Manufatura \\
Design for Modularity & Projeto para Modularidade \\
Design for Packaging & Projeto para Embalagem \\
Design for Precision & Projeto para Precisão \\
Design for Reliability & Projeto para Confiabilidade \\
Design for Robustness & Projeto para Robustez \\
Design for Safety & Projeto para Segurança \\
Design for Standards & Projeto para Normalização \\
Design for Supportability & Projeto para Apoio Logístico \\
Design for Testability & Projeto para Teste \\
Design for Ergonomics & Projeto para Ergonomia \\
\hline
\end{tabular}

Fonte: Adaptado de Back et al. (2008) 
Entre as metodologias mais conhecidas estão o Design for Manufacturing (DfM) e o Design for Assembly (DfA), que, quando aplicadas simultaneamente, formam o DfMA - Design for Manufacturing and Assembly, que tem como objetivo revisar o projeto do produto considerando a capacidade de fabricação da empresa, isto proporciona facilidade de fabricação, bem como redução do custo dos produtos, refletindo diretamente na melhoria da qualidade do produto (BOOTHROYD, 2001; ESTORILIO \& SIMIÃO, 2006; HARIK; SAHMRANI, 2010).

De acordo com Barbosa (2012), Design for Reliability, ou Projeto para Confiabilidade, é uma metodologia para melhoria do produto que está diretamente ligada à sua quantidade de componentes, ou seja, quanto menos componentes, menor será a probabilidade de o produto falhar. Assim, o projeto do produto deve ser eficiente a fim de prevenir falhas, e isso pode ser atingido minimizando a quantidade de componentes. Bralla (1996) apresenta algumas características que, quando aplicadas ao projeto, podem auxiliar na confiabilidade do produto, dentre as quais se pode destacar:

- Simplicidade: os produtos devem ter o menor número de peças;

- Padrões de materiais e componentes: utilizar componentes disponíveis no mercado;

- Projeto padronizado do próprio produto: quando vários produtos semelhantes estão para ser produzidos, é necessário especificar os mesmos materiais, peças e subconjuntos para cada um deles;

- Tolerâncias mais amplas: tolerâncias mais apertadas trazem custos mais elevados;

- Uso de materiais mais processáveis: utilizar os materiais mais processáveis disponíveis considerando as características funcionais do produto;

- Prevenção de operações secundárias: projetar para eliminar ou simplificar as operações de fabricação e montagem;

- Utilizar características especiais dos processos: conhecer as características especiais dos processos de fabricação e utilizar esses recursos para eliminar operações desnecessárias.

\section{Metodologia}

A metodologia aplicada neste trabalho caracteriza-se por meio de pesquisa tecnológica, que busca a geração de produtos, com base em um estudo de caráter qualitativo e quantitativo, baseando-se em revisão da literatura e posterior estudo de caso exploratório. Para o desenvolvimento do produto foram utilizadas algumas etapas do modelo apresentado por Pahl et al. (2013), em que são desenvolvidas as etapas de projeto conceitual, projeto detalhado e, por fim, a prototipagem e os testes iniciais do produto, conforme mostra a Figura 1.

Figura 1 - Etapas de desenvolvimento de produto adotado neste trabalho.

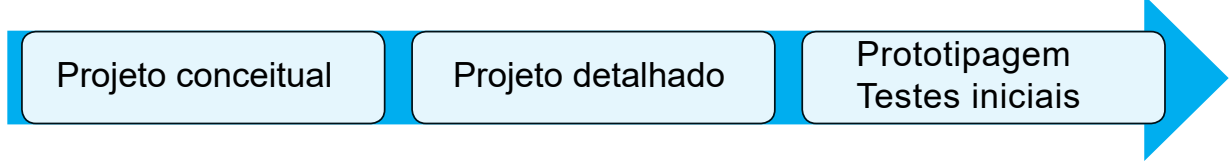

Fonte: Elaborado pelos autores, 2020.

Inicialmente, foi realizado o projeto conceitual, no qual são apresentadas as diretrizes para o projeto. Nessa etapa é descrito o conceito do produto, bem como são apresentados seus componentes e montagens necessárias. Posteriormente, no projeto detalhado, é realizada a descrição específica de cada componente, sendo especificados os materiais utilizados. Por fim, é realizada análise criteriosa baseada em testes e ensaios para validar e aprovar a construção do produto.

\section{Desenvolvimento}

Neste tópico serão apresentadas as informações referentes às etapas aplicadas ao processo de desenvolvimento e teste do produto, bem como as ferramentas utilizadas para auxiliar o projeto do dispositivo. 


\subsection{Projeto conceitual}

Nesta etapa foram levantadas as informações referentes às necessidades do dispositivo, bem como aos requisitos para o projeto do produto. Esses dados foram pesquisados diretamente com técnicos e profissionais que fazem uso de dispositivos de fixação, sendo que tais informações foram alinhadas às diretrizes de Bralla (1996) a fim de otimizar o projeto do produto. A Tabela 2 apresenta algumas características observadas que são essenciais para o equipamento e devem ser priorizadas na etapa de projeto para garantir a confiabilidade do dispositivo.

Tabela 2 - Requisitos essenciais para o projeto do produto.

\begin{tabular}{ll}
\hline Requisitos do projeto & Descrição \\
\hline Garantir a fixação & $\begin{array}{l}\text { Garantir a fixação das peças a serem manufaturadas, bem como utilizadas em sistemas de } \\
\text { medição; }\end{array}$ \\
Repetibilidade & Garantir a repetibilidade em casos de refixação das peças; \\
Minimizar componentes & Projetar um número mínimo de componentes; \\
Confiabilidade & Desenvolver alternativas para agilizar o processo e manter a confiabilidade do dispositivo; \\
Projetar tolerâncias & $\begin{array}{l}\text { Avaliar a necessidade dos níveis de tolerâncias geométricas utilizadas no dispositivo (circularidade } \\
\text { e posição); }\end{array}$ \\
Acessórios de fixação & $\begin{array}{l}\text { Eliminar a necessidade de utilização de ferramentas manuais ou acessórios de fixação para } \\
\text { manuseio do dispositivo; }\end{array}$ \\
Intercambialidade & $\begin{array}{l}\text { Capacidade de ser utilizado em diversas máquinas operatrizes e de medição ou controle de } \\
\text { geometria. }\end{array}$ \\
\hline
\end{tabular}

Fonte: Elaborado pelos Autores, 2020.

Com base nessas informações, pode-se iniciar o processo de concepção do conceito. Essa atividade foi realizada com auxílio de software CAD 3D, especificamente o SolidWorks ${ }^{\circledR}$. Assim, foi possível criar uma imagem visual do dispositivo, conforme apresentado na Figura 2. Pode-se observar que o projeto foi dividido em três módulos:

- Base: peça única composta por uma chapa metálica, na qual se localiza a área de esquadrejamento do dispositivo;

- Trava excêntrica: conjunto montado e dotado de um dispositivo excêntrico que executa a fixação das peças;

- Grampo de fixação: conjunto montado a ser manufaturado, consistindo em um sistema de fixação auxiliar para esforços no sentido normal à base.

Figura 2 - Projeto conceitual do dispositivo - Módulos.

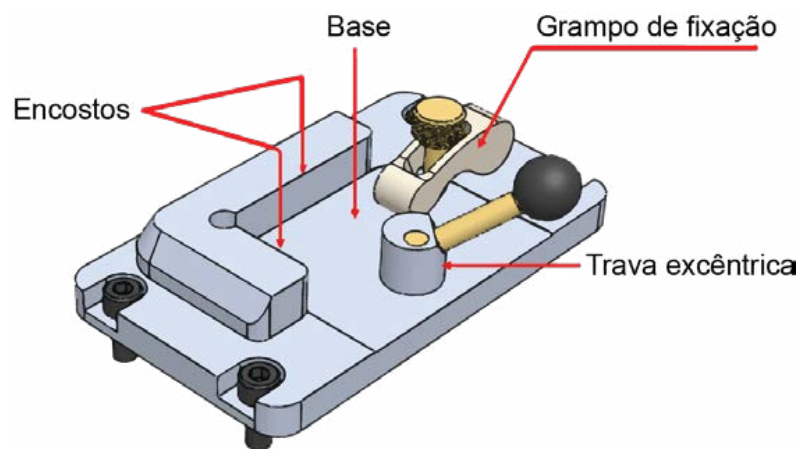

Fonte: Elaborado pelos autores, 2020.

A base do dispositivo foi projetada para garantir o esquadrejamento das peças nela fixadas. Os encostos (dois), em formato ortogonal, foram desenvolvidos com elevado controle de geometria, em que o erro de 
perpendicularidade é de $0,002 \mathrm{~mm} \pm 2 \mu \mathrm{m}$, bem como a planicidade da superfície, que entra em contato com as peças, deve possuir erro de $0,002 \mathrm{~mm} \pm 2 \mu \mathrm{m}$.

O conjunto de trava excêntrica possui um sistema de fixação capaz de posicionar a peça com apenas um movimento menor ou igual a $90^{\circ}$, o que a direciona contra as duas paredes de esquadrejamento da base, mantendo-a nessa posição. Depois de realizada a trava da peça, o operador precisa acionar o grampo de fixação para garantir seu posicionamento e evitar que, por algum motivo, ela possa ser forçada a perder sua posição no sentido normal à base. Com esses sistemas, espera-se alcançar os requisitos essenciais de confiabilidade e repetibilidade para o produto, uma vez que se torna à prova de erros do operador, sendo que o movimento por rotação excêntrica garante a posição pelo seu terceiro ponto de contato. É importante se destacar que a fixação das peças no dispositivo é realizada sem a necessidade de ferramentas, ou seja, o processo é completamente manual.

A Figura 3 apresenta a sequência operacional a ser realizada para a fixação de uma peça no dispositivo. Inicialmente, o operador posiciona a peça sobre a base (Ver Figura 3a); na sequência, ele aciona a alavanca da trava excêntrica, que automaticamente direciona a peça contra as paredes da base, realizando seu esquadrejamento (Ver Figura 3b). Por fim, deve ser realizado o acionamento do grampo de fixação que irá garantir a precisão do processo (Ver Figura 3c).

Figura 3 - Sequência operacional de utilização do dispositivo.

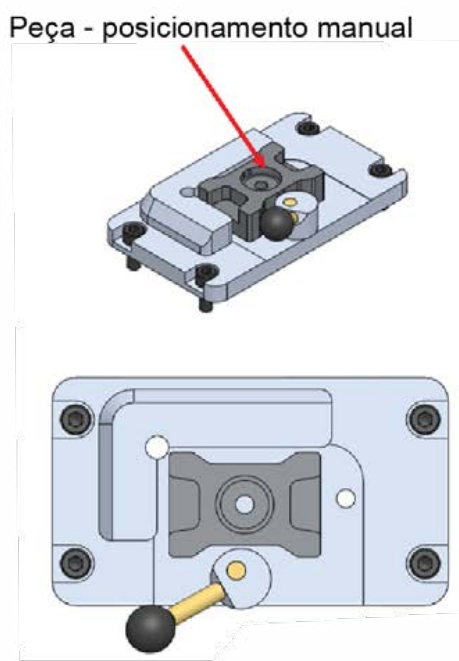

a)

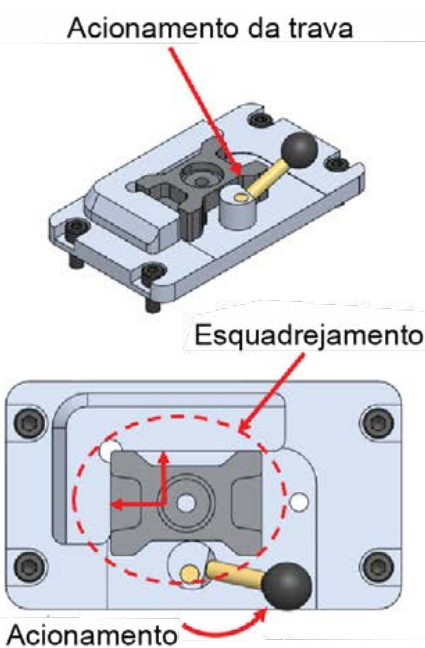

b)

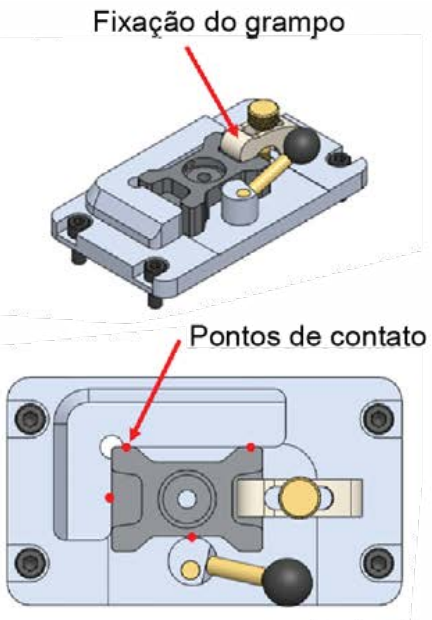

c)

Fonte: Elaborado pelos autores, 2020.

\subsection{Projeto detalhado}

Nesta etapa do projeto são definidas as características relacionadas às dimensões e à geometria do produto, bem como a definição de todos os itens que irão compor sua montagem. Na Figura 4 é apresentada uma vista explodida da montagem dos módulos, na qual se observa todos os componentes que compõem o produto, bem como os parafusos utilizados para sua fixação. 
Figura 4 - Sequência operacional de montagem do dispositivo.

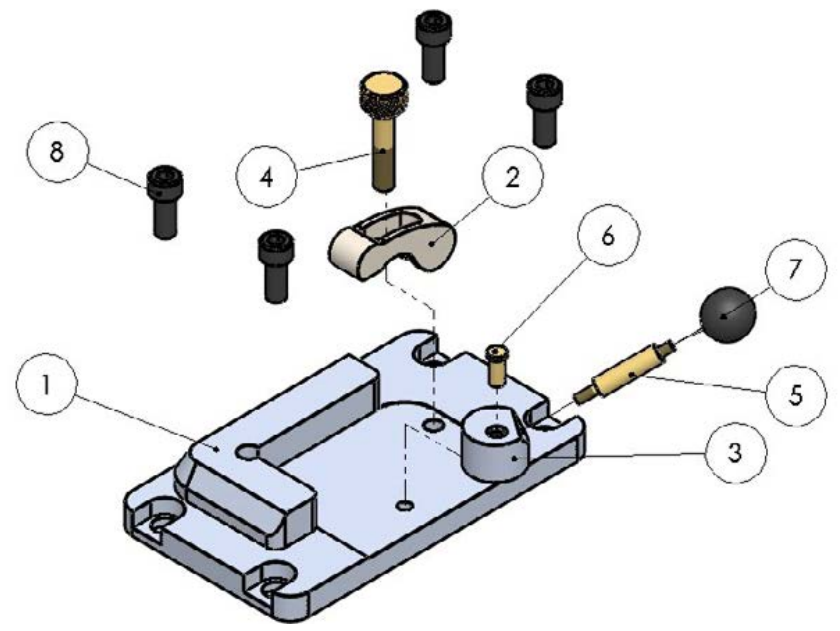

Fonte: Elaborado pelos autores, 2020.

As informações referentes a cada peça são mostradas na Tabela 3, que apresenta uma lista de materiais, contendo detalhes dos componentes do dispositivo e a descrição de cada item em termos de quantidade, material e processo de manufatura utilizado na sua fabricação. Conforme se pode observar, o produto montado possui apenas 11 peças, incluindo os parafusos de fixação, ou seja, procurou-se minimizar a quantidade de componentes do dispositivo sem perda de função dos módulos, o que corrobora com as diretrizes de Bralla (1996) para otimização de produtos. Outro ponto importante a se destacar se refere aos componentes adquiridos de terceiros (peças 7 e 8), sendo facilmente encontrados no mercado.

Tabela 3 - Lista de materiais.

\begin{tabular}{lllll}
\hline & Peça & Qtde. & \multicolumn{1}{c}{ Material } & \multicolumn{1}{c}{ Processo de manufatura } \\
\hline 1 & Base principal & 1 & Ferro fundido nodular & Usinagem CNC, tratamento superficial e retificação \\
2 & Trava do grampo & 1 & Aço 1045 & Usinagem CNC \\
3 & Eixo excêntrico & 1 & Aço 1045 & Usinagem CNC e tratamento superficial \\
4 & Parafuso do grampo & 1 & Aço 1045 & Usinagem CNC \\
5 & Eixo do manipulo & 1 & Aço 1020 & Usinagem CNC \\
6 & Pino trava & 1 & Bronze TM23 & Usinagem \\
7 & Esfera do manipulo & 1 & Polipropileno & $*$ \\
8 & Parafuso M8 & 4 & Aço liga & $*$ Temperado e revenido \\
\hline
\end{tabular}

* Componente disponível no mercado

Fonte: Elaborado pelos autores, 2020.

$\mathrm{Na}$ Figura 5 é apresentado o desenho técnico da base do dispositivo (peça n. ${ }^{0} 1$ ), em que se observa seu detalhamento dimensional parcial, suas medidas e detalhes de construção. Na Figura 6 são apresentados os desenhos técnicos dos outros componentes (peças n. ${ }^{\circ} 2,3,4,5$ e 6 ). 
Figura 5 - Desenho técnico - base de dispositivo, peça 1.
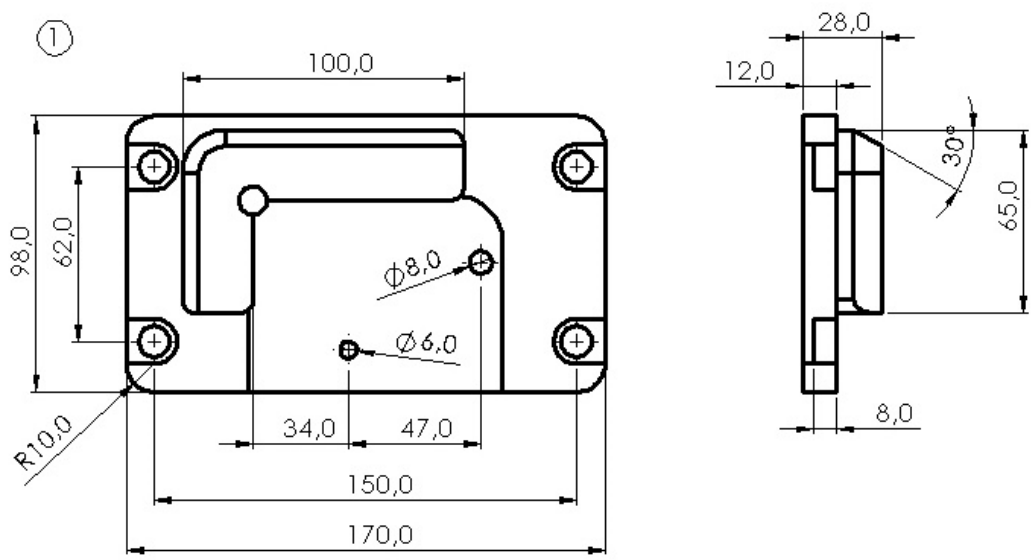

Fonte: Elaborado pelos autores, 2020.

Figura 6 - Desenho técnico - peças 2, 3, 4, 5 e 6.

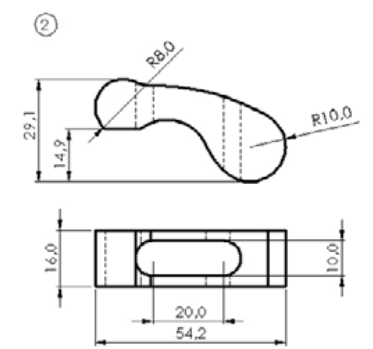

(5)

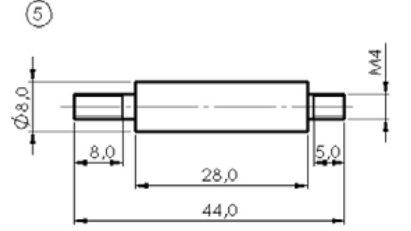

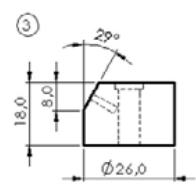
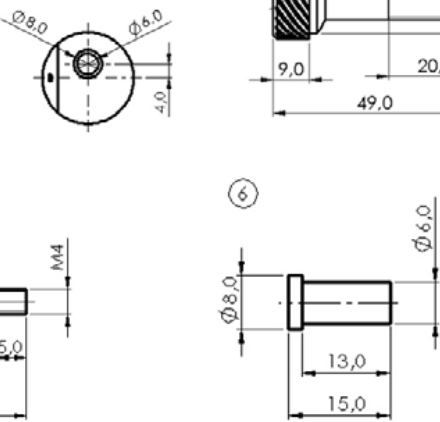

Fonte: Elaborado pelos autores, 2020.

\subsection{Prototipagem e testes iniciais}

O dispositivo apresentado no item 4.2 foi produzido, sendo que suas peças foram usinadas num processos de usinagem CNC (torneamento e fresamento) e, posteriormente, montadas conforme mostra a Figura 7, que apresenta uma comparação entre conceito e produto desenvolvido. Convém ressaltar que nessa etapa alguns dos materiais projetados foram substituídos a fim de reduzir os custos. Por se tratar de um protótipo, os componentes base, trava do grampo e eixo excêntrico tiveram seus materiais alterados por alumínio, isto por questões de facilidade de fabricação, porém não se recomenda essa troca para comercialização do dispositivo, pois pode comprometer sua durabilidade. 
Figura 7 - Comparação entre conceito do produto e protótipo.

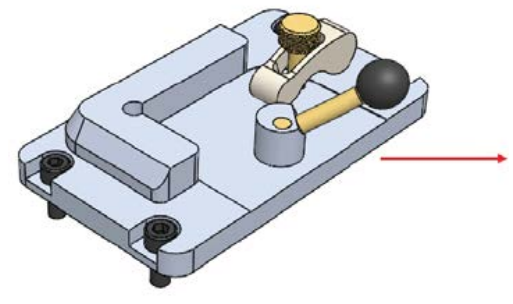

Projeto conceitual

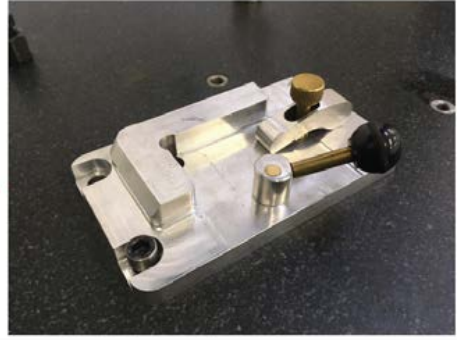

Protótipo

Fonte: Elaborado pelos autores, 2020.

O protótipo construído foi fixado em alguns equipamentos de usinagem e medição para fins de avaliação posicional, conforme se pode observar na Figura 8. Na Figura 8a, o dispositivo se localiza na placa de um torno convencional, em que ele pode ser utilizado para usinagem por várias operações, assim como na Figura $8 \mathrm{~b}$ e $8 \mathrm{c}$, em que ele foi fixado sobre a mesa de uma fresadora e uma máquina de usinagem por eletroerosão, respectivamente. Na Figura 8d o dispositivo se encontra localizado na mesa de desempeno de uma máquina de medir por coordenadas, em que ele pode ser utilizado para realizar medições e controle geométrico de peças.

Figura 8 - Posicionamento do dispositivo em máquinas/equipamentos.

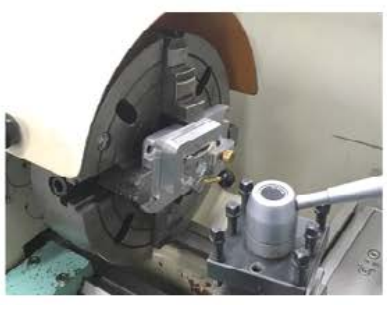

a)

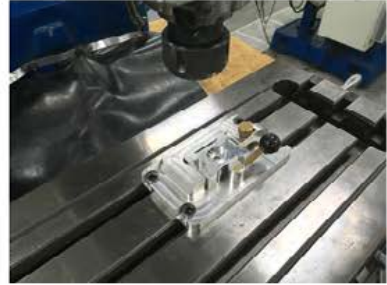

b)

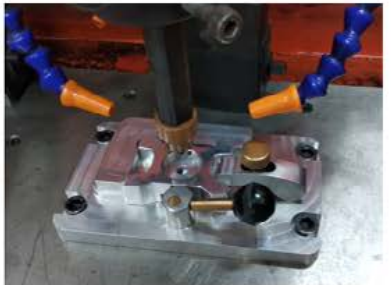

c)

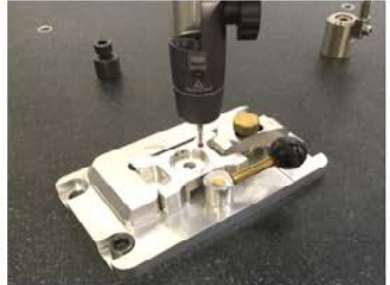

d)

Fonte: Elaborado pelos autores, 2020.

Para a validação do dispositivo foi realizada a usinagem por meio de eletroerosão de três amostras com diâmetros internos bipartidos e reposicionados três vezes para cada operação, conforme se pode observar na Figura 9a. Esta foi submetida à verificação dos erros geométricos de forma. Nessa etapa foi utilizado um medidor de circularidade e cilindricidade do fabricante Mitutoyo ${ }^{\circledR}$, modelo Roundtest RA-1600, conforme mostra a Figura $9 \mathrm{~b}$, na qual se observa o processo de medição aplicado. Esse sistema possui uma mesa giratória, em que a peça é posicionada com exatidão rotacional de $0,02+6 \mathrm{H} / 10000 \mu \mathrm{m}$ e axial de $0,02+6 \mathrm{X} / 10000 \mu \mathrm{m}$, com sistema Digital Adjustment Table (DAT), que elimina o erro humano resultante de possíveis colisões.

Figura 9 - Processo de medição - medidor de circularidade e cilindricidade.

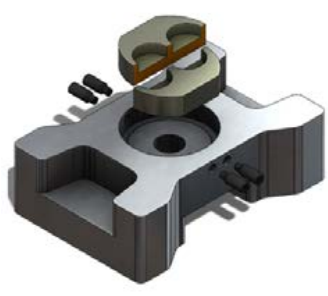

a) Peça de teste

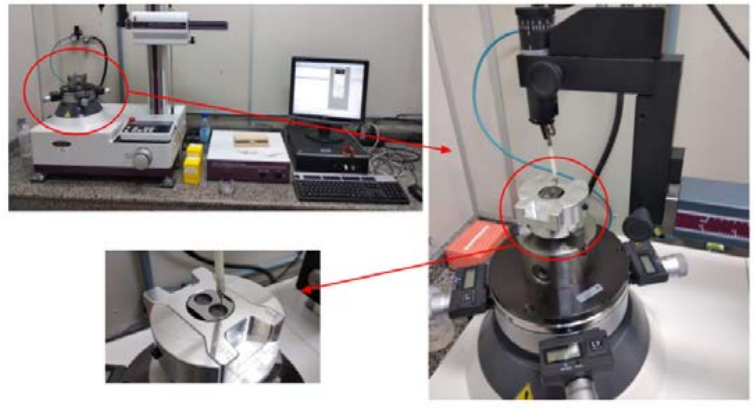

b) Processo de medição

Fonte: Elaborado pelos autores, 2020. 
Foi utilizado um cut off de filtragem em 50 ondulações por revolução (UPR), conforme recomendado no manual de instruções do medidor de circularidade, observando os campos dimensionais das amostras. Todas as medições foram realizadas com apalpador em rubi com diâmetro de $1 \mathrm{~mm}$. Para a interface computacional foi aplicado o software Roundpak®, que permite a plotagem de gráficos para melhor compreensão das medições. A partir dessas características de construção do equipamento, verifica-se uma elevada sensibilidade aos erros resultantes do processo de usinagem.

A Tabela 4 apresenta os resultados das medições, em que são descritos os dados para as três peças medidas, sendo possível observar que a média da cilindricidade obtida foi de 12,232 $\mu \mathrm{m}$, que se trata de um valor relevante para processos de usinagem de componentes industriais em geral.

Tabela 4 - Resultados das medições.

\begin{tabular}{ccc}
\hline Peça & Cilindricidade & Unidade \\
\hline 1 & 12,394 & $\mu \mathrm{m}$ \\
2 & 11,278 & $\mu \mathrm{m}$ \\
3 & 13,024 & $\mu \mathrm{m}$ \\
Média & 12,232 & $\mu \mathrm{m}$ \\
\hline
\end{tabular}

Fonte: Elaborado pelos autores, 2020.

Nas Figuras 10, 11 e 12 são apresentados os gráficos do relatório da avaliação de cilindricidade gerados pelo software do equipamento de medição para as peças 1, 2 e 3, respectivamente. Nessas figuras, pode-se observar a distribuição dos pontos no processo e o comportamento associado à cilindricidade das cavidades usinadas. Pode-se notar, também, a disposição das curvas e seu comportamento de acordo com o perfil de medição.

Figura 10 - Gráficos referentes ao resultado da medição de cilindricidade para peça 1.
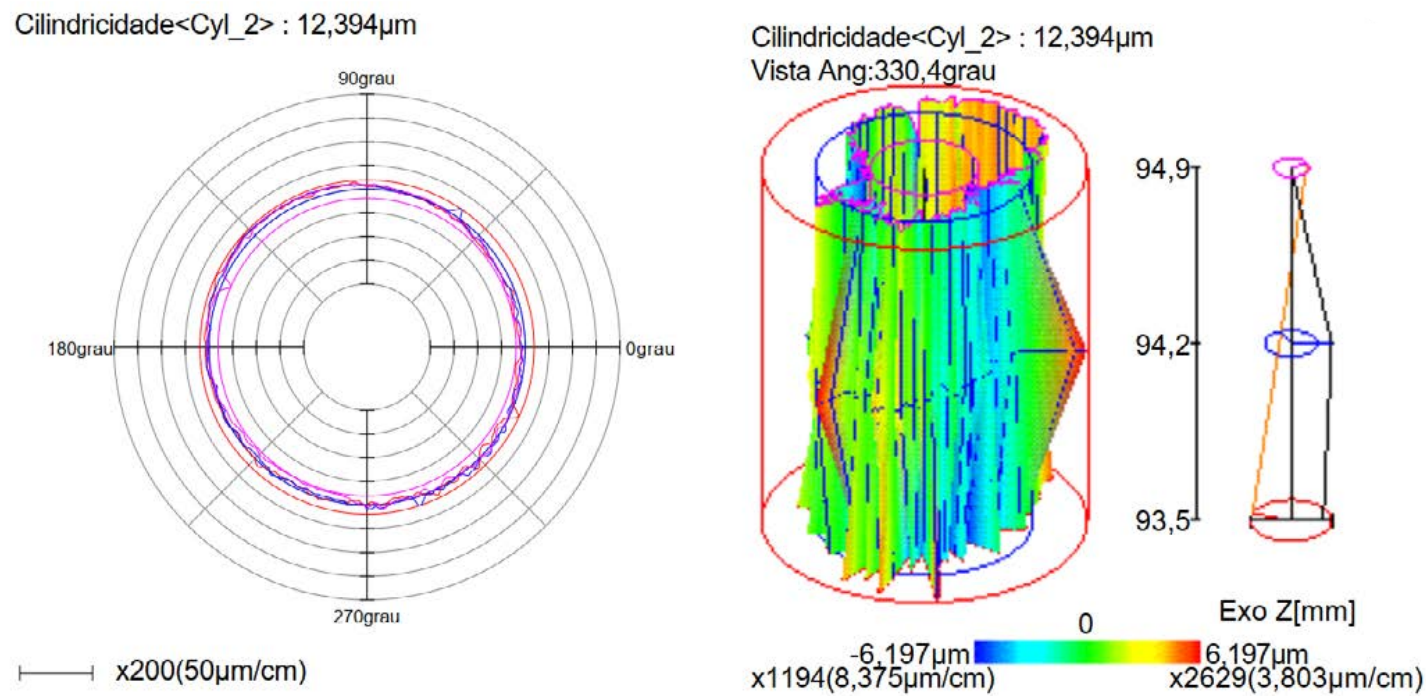

Fonte: Elaborado pelos autores, 2020. 
Figura 11 - Gráficos referentes ao resultado da medição de cilindricidade para peça 2.
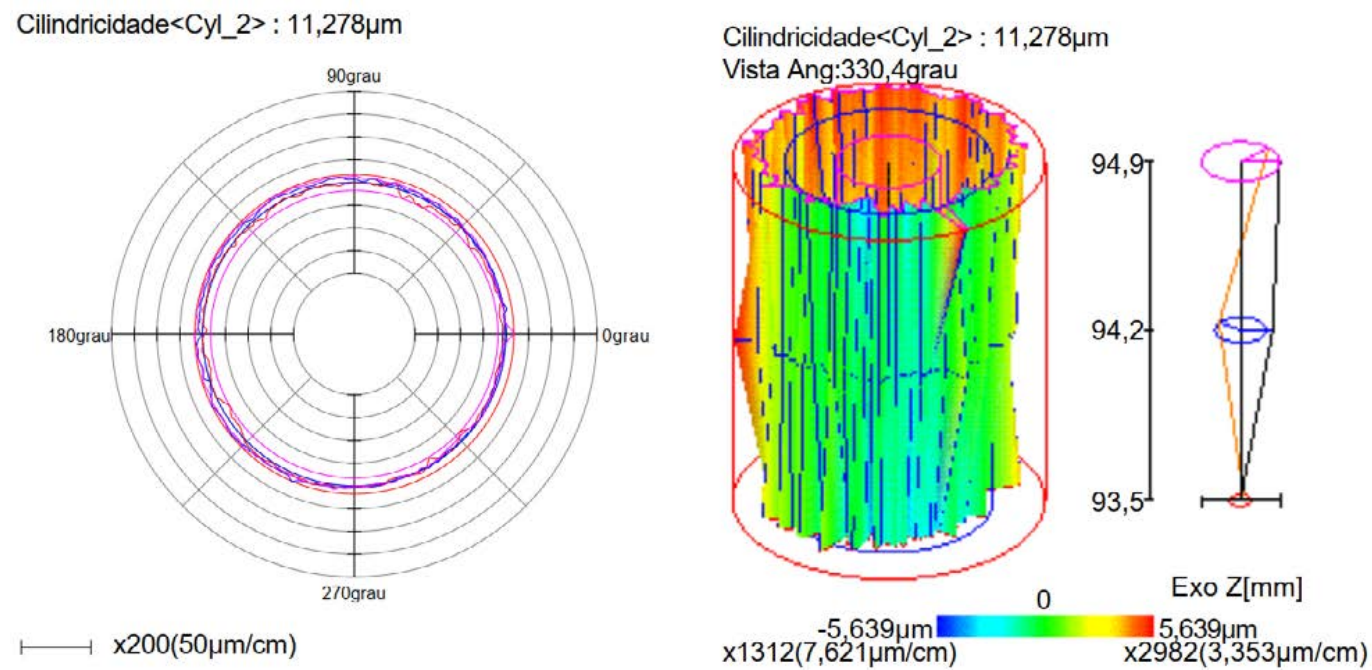

Fonte: Elaborado pelos autores, 2020.

Figura 12 - Gráficos referentes ao resultado da medição de cilindricidade para peça 3.
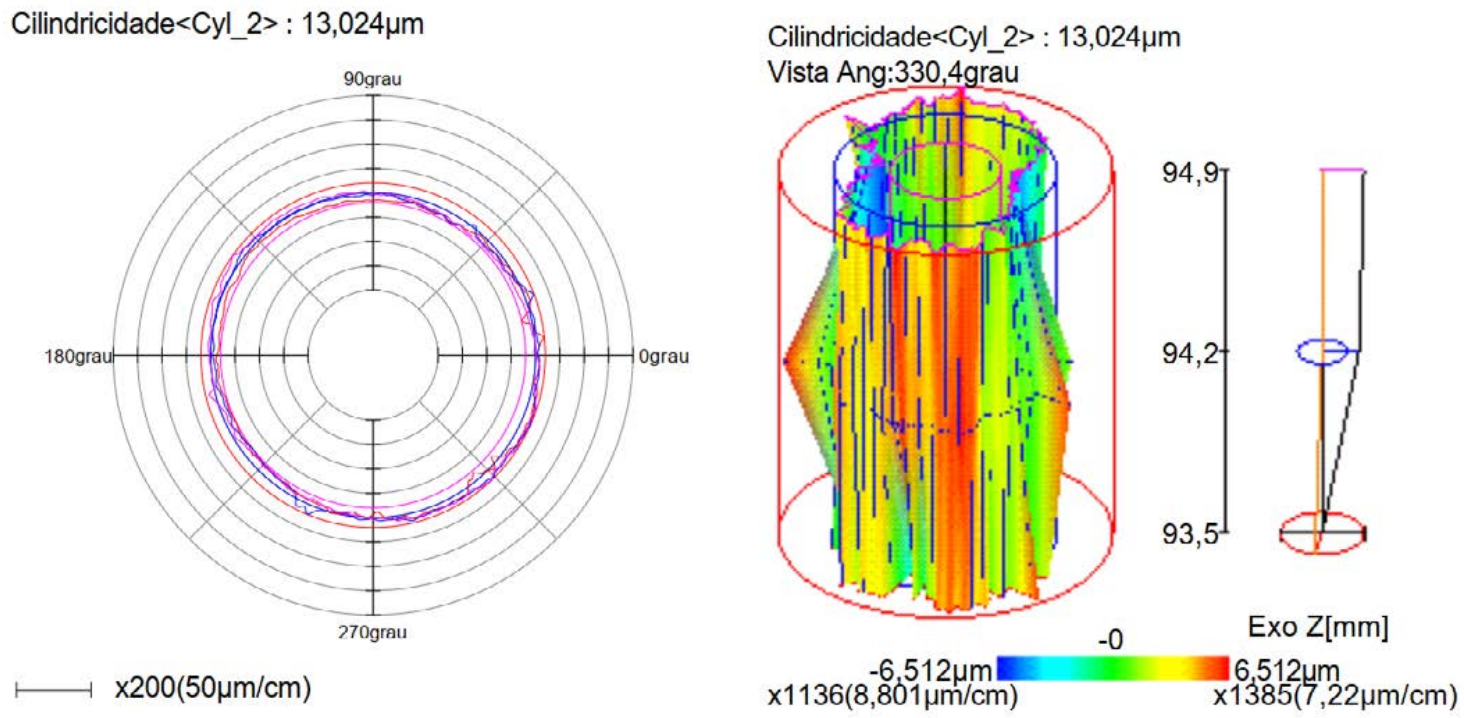

Fonte: Elaborado pelos autores, 2020.

\section{Considerações finais}

Este trabalho utilizou a metodologia de desenvolvimento de produto alinhada ao conceito de DfX (Projeto para Excelência) para buscar o conceito de um equipamento adequado às necessidades de fixação para processos de usinagem ou similares.

Diante dos resultados apresentados, conclui-se que o objetivo deste trabalho foi atendido, uma vez que o equipamento desenvolvido se apresentou viável para sua finalidade proposta. Foram apresentadas as etapas do desenvolvimento do produto, suas características, os materiais utilizados e uma breve descrição do processo de montagem. Por fim, foram realizados testes, nos quais foi possível observar os efeitos do processo de fixação nas tolerâncias geométricas de circularidade. 
Pode-se ponderar que a média de 12,232 $\mu \mathrm{m} \pm 0,792 \mu \mathrm{m}$ obtida com a avaliação da circularidade é um resultado fortemente positivo, uma vez que, para processos dessa natureza, são consideradas grandezas de baixa incerteza, o que valida o desenvolvimento e construção do dispositivo.

Por fim, como sugestões, serão realizadas otimizações no produto para promover sua intercambialidade, especificamente alterações no sistema de fixação, de modo que se sugere criar oblongos para ampliar a margem de posições de fixação nos equipamentos em que ele é utilizado e para futuras posições que possam ocorrer a partir de sua necessidade.

A partir dos resultados deste trabalho, espera-se contribuir para aprimorar o processo de usinagem em máquinas operatrizes, uma vez que o projeto do produto se encontra finalizado e testado. Este pode ser produzido e utilizado como alternativa viável de fixação de peças, com garantia de que seu funcionamento irá atender às necessidades dimensionais e geométricas de peças, seja por meio de máquinas operatrizes, seja por meio de equipamentos de medição, seja por outros processos de fabricação.

\section{Referências}

BARBOSA, G. F. Desenvolvimento de um modelo de análise para implantação de automação na manufatura aeronáutica. 2012. 332 f. Tese de Doutorado - Curso de Engenharia Mecânica, Departamento de Engenharia Mecânica, Universidade de São Paulo, São Carlos, 2012.

BOOTHROYD, G.; DEWHURST, P.; KNIGHT, W. Product design for manufacture and assembly. New York: Marcel Dekker, 2001.

BRALLA, J. G. Design for excellence. New York: McGrawHill, 1996. 326 p.

BUSS, C. O.; CUNHA G.D. Modelo Referencial para o Processo de Desenvolvimento de Novos

Produtos. In: XXII Simpósio de Gestão da Inovação Tecnológica, Salvador, 2002.

ESTORILIO, C.; SIMIÃO, M. C. Cost reduction of a diesel engine using the DFMA method. Product Management \& Development, v. 4, n. 2, p. 95-103, 2006.

HARIK, R. F.; SAHMRANI, N. DFMA+, A Quantitative DFMA Methodology, Computer-Aided Design and Applications, v. 7, n. 5, p. 701-709. 2010. DOI https://doi.org/10.3722/cadaps.2010.701-709

IVANOV, $\mathrm{V}$. et al. Determination of contact points between workpiece and fixture elements as a tool for augmented reality in fixture design. Wireless Networks, v. 2, 2019. DOI: https://link.springer.com/article/10.10 07\%2Fs11276-019-02026-2

IVANOV, V.; ZAJAC, J. Flexible Fixtures for CNC Machining Centers in Multiproduct Manufacturing. EAI Endorsed Transactions on Industrial Networks and Intelligent Systems, v. 4, n. 12, p. 153552, 2018. DOI: https://eudl.eu/doi/10.4108/eai.10-1-2018.153552

KARPUS, V. et al. Technological assurance of complex parts manufacturing. Springer International Publishing. ISBN: 9783319935874. 2019. 51-61 p.

LIU, Y.; LI, Y.; WEN, X. Modeling for locating pin adjustment on fixtures to improve positioning accuracy of parts. Proceedings of the 2018 IEEE International Conference on Advanced Manufacturing, ICAM 2018, p. 61-64, 2019. DOI: https://doi.org/10.1109/AMCON.2018.8615050

MEDEIROS, E. N. Uma proposta de metodologia para o desenvolvimento de projeto de produto. (Dissertação, Mestrado em Engenharia de Produção). - COPPE/UFRJ, Rio de Janeiro: 1981.

MENDES, H. et al. Smart Design and Manufacturing of Power Transformers Tanks. Proceedings - 2019 IEEE International Conference on Environment and Electrical Engineering and 2019 IEEE Industrial and Commercial Power Systems Europe, EEEIC/I and CPS Europe 2019, 2019. DOI: https://doi.org/10.1109/ EEEIC.2019.8783902 
PAHL, G.; BEITZ, W.; FELDHUSEN, J.; GROTE, K.-H. Projeto na engenharia: Fundamentos do desenvolvimento eficaz de produtos, métodos e aplicações. 6. ed. São Paulo: Edgard Blucher, 2013.

ROZENFELD, H; FORCELLINI, F. A.; AMARAL, D. C.; TOLEDO, J. C; SILVA, S. L; ALLIPRANDINI, D. H.; SCALICE, R. K. Gestão de desenvolvimento de produtos: uma referência para a melhoria do processo. São Paulo: Saraiva, 2006.

TZIMAS, E.; VOSNIAKOS, G. C.; MATSAS, E. Machine tool setup instructions in the smart factory using augmented reality: a system construction perspective. International Journal on Interactive Design and Manufacturing, v. 13, n. 1, p. 121-136, 2019. DOI: https://doi.org/10.1007/s12008-018-0470-z

ZHAO, Q. Application of Intelligent Systems in Multi-modal Information Analytics. Springer International Publishing. ISBN: 978-3-030-15738-8. 2019. 596-603 p.

\title{
Sobre os autores
}

\begin{abstract}
Cassiano Rodrigues Moura
Mestre em Ciências e engenharia de materiais pela Universidade do Estado de Santa Catarina (Udesc), graduado em Engenharia de produção e sistemas (UDESC). Pós-graduado em Logística. Pós-graduado em Engenharia de produção. Professor do Instituto Federal de Santa Catarina (IFSC), coordenador de cursos FIC, alocado no Jaraguá do Sul - Rau. Tem experiência nas áreas de mecânica e engenharia de produção, com ênfase em desenvolvimento de produtos.
\end{abstract}

\section{Giovani Conrado Carlini}

Doutorando em Engenharia mecânica na Pontifícia Universidade Católica do Paraná (Puc-PR), área de concentração: fabricação. Possui mestrado em Engenharia mecânica na Universidade Federal de Santa Catarina (UFSC), área de concentração: fabricação, e graduação em Tecnologia em processos de produção mecânica (Senai - Departamento Regional de Santa Catarina). Tem experiência na área de engenharia mecânica e industrial, atuando principalmente nos processos de usinagem e processos por remoção térmica. Atualmente é professor na área de fabricação mecânica do Instituto Federal de Educação, Ciência e Tecnologia de Santa Catarina (IFSC).

\section{Cristiano da Silva}

Doutorando em Ciência e Engenharia de materiais pela Universidade do Estado de Santa Catarina(Udesc). Possui formação em curso Técnico em Mecânica (2001) pela Escola Técnica Tupy/SOCIESC, graduação em Tecnologia mecânica (2010) pela Udesc e mestrado em Engenharia e ciência de materiais pela Universidade Estadual de Ponta Grossa (UEPG) (2014). Atualmente, é professor EBTT do Instituto Federal de Educação, Ciência e Tecnologia de Santa Catarina (IFSC), campus Jaraguá do Sul - Rau.

Recebido em: 10.03 .2020

Aceito em: 04.03 .2021 